\title{
The UDWT image denoising method based on the PDE model of a convexity- preserving diffusion function
}

\author{
Xianghai Wang ${ }^{1,2^{*}}$, Wenya Zhang ${ }^{3}$, Rui $\mathrm{Li}^{3}$ and Ruoxi Song ${ }^{1 *}$
}

\begin{abstract}
It is a great challenge to maintain details while suppressing and eliminating noise of the image. Considering the nonconvexity property of the diffusion function and the hypersensitivity of the Laplace operator to noise in the $\mathrm{Y}-\mathrm{K}$ model, a fourth-order PDE image denoising model (Con_G\&L model) is proposed in this paper. This model is constructed by a new convexity-preserving diffusion function which guarantees the corresponding energy functional has a globally unique minimum solution. At the same time, the Gaussian filter is combined with the Laplace operator in this model, and as a result, the noisy image is smoothed before the diffusion process, which improves the ability of capturing the details and edges of the noisy image greatly. Furthermore, by analyzing the statistical properties of the undecimated discrete wavelet transform (UDWT) coefficients of noisy image, we observe that the noise information is mainly distributed in the high-frequency sub-bands, and based on this, the proposed Con_G\&L model is applied in the high-frequency sub-bands of the UDWT to get the denoising method. The proposed method removes the image noise effectively with the image texture and other details of the image being maintained. Meanwhile, the generation of false edges and the staircase effect can be suppressed. A large number of simulation experiments verify the effectiveness of the proposed method.
\end{abstract}

Keywords: Undecimated discrete wavelet transform (UDWT), Fourth-order partial differential equations, Diffuse function, Convexity-preserving, Image denoising

\section{Introduction}

In the process of image formation and transmission, some noise will be introduced, having a great impact on the subsequent applications. Therefore, effectively suppressing and eliminating noise in images has always been a popular research topic in the image processing area. Several of image modeling methods have been proposed to study the relationship between the image background and the noise component [1-3]. Generally, a good denoising method should be able to remove the noise from the image while maintaining the information of the edges, contours, and details of the image. In other words, it should remove noise and retain the spatial resolution of the images at the same time.

In recent years, the partial differential equation (PDE) method has become one of the most important

\footnotetext{
* Correspondence: xhwang@|nnu.edu.cn; ruoxisong@qq.com

'School of Geography, Liaoning Normal University, Dalian 116029, China Full list of author information is available at the end of the article
}

mathematical tools for image modeling and representation due to its good property of flexibility and local adaptability. Based on the continuous mathematical image model, this kind of method makes the image follow a specified $\mathrm{PDE}$, the processing result of which is considered the expected result [4-6]. In the field of image denoising, the second-order PDE nonlinear diffusion equation (P-M model) proposed by Perona and Malik is a pioneer method in PDE image denoising [7]. This model combines image denoising with edge detection organically and takes into account the preservation of details while denoising. However, in the process of iteration, this model is unbounded for the boundary detector oscillation when large noise is introduced, and the condition given by the model is smooth, which will affect the results. Also, the model is ill-posed. To tackle these, Alvarez et al. proposed regularized P-M model [8]. However, the regularized model is not stable when the difference is zero in the diffusion process. Additionally, the diffusion degree is weakened by 
the first-order partial differential at the edge and texture regions because of the second-order characteristics of the model, and after several iterations, the image gray level will appear as a piecewise constant, and the "block" effect will make it difficult to retain the texture details of the original image. To solve these problems, researchers ought to find a PDE model with higher order. The fourth-order PDE model is noted for its stability and computational efficiency. The Y-K model proposed by You and Kaveh in [9] and the LLT model proposed by Lysaker in [10] are some typical models. They efficiently suppress the "block" effect introduced by the second-order PDE model. However, the Y-K model causes an obvious gray difference between some points and their surroundings. In addition, black and white outliers usually emerge in the denoised image. The reason is that the Laplace operator in the model is sensitive to speckle noise, which restrains the diffusion of the model. The LLT model is based on the minimum L1 norm about the second derivative of the image, and there is a fast computational method of numerical solution [11]. Nevertheless, it is essentially a high-order filter, which is more sensitive to the high-frequency information of the image and will inevitably blur the image details and edge information with the diffusion deepening.

The wavelet transform is an effective tool for timefrequency analysis of signals due to its excellent timefrequency localization ability. Therefore, image denoising methods based on wavelets have frequently been considered. The most typical methods are based on a wavelet domain, including the general threshold method [12], extreme threshold method [13], St. Ein unbiased risk threshold method [14], and Bayesian threshold method [15-17]. These methods simply and rapidly obtain the corresponding denoising thresholds by using the frequency characteristics of decomposing the sub-band coefficients through image wavelets. However, these methods either have the tendency of "over-stifling" [12, 13] or "over-reserving" [14] the wavelet coefficients. The accuracy of the Bayesian threshold method in estimating the variance of sub-band noise remains to be improved.

Based on the above discussions, in this paper, a novel image denoising method is proposed by the improved Y$\mathrm{K}$ model and UDWT. First, a novel convexity-preserving diffusion function is proposed by introducing the Gaussian convolution process to the traditional Y-K model, which ensures the unique minimum solution of the model and decreases the sensitivity of the Laplace operators with respect to speckle noise. Then, the statistical property of noisy image UDWT coefficients is studied; we observe that the noise information is mainly distributed in the high-frequency sub-bands, and based on this, the proposed Con_G\&L model is applied in the highfrequency sub-bands of the UDWT to get the denoising method. A large number of experiments are carried out to verify the effectiveness of the proposed method. The results show that the proposed method can effectively remove the noise in the image while retaining the image edge, texture, and other information details.

The rest of the paper is organized as follows: Section 2 provides the statistical analysis of UDWT coefficient and gives the proposed denoising model, Section 3 gives the description of the datasets and the details of the experiments, and Section 4 presents the conclusions.

\section{Methods}

\subsection{Statistical analysis of UDWT coefficients of noisy images}

The traditional discrete wavelet transform can decompose an image to multi-scales and multi-directions. However, due to the downsampling and upsampling process, the wavelet transform is not shift-invariant. To solve this, the undecimated discrete wavelet transform (UDWT) was proposed by Shensa [18], which not only retains the properties of wavelet transform, but also has the good property of shift-invariant. UDWT does not utilize the downsampling operation in the process of signal decomposition, and instead, it inserts zeroes every two coefficients to expand the filter in the process of high-pass and low-pass filtering. The length of lowfrequency and high-frequency signals obtained by UDWT decomposition is the same as that of the original signal, which not only preserves the time-frequency local characteristics and multiresolution analysis characteristics of the wavelet transform but also ensures preservation of the translation invariant characteristics. These characteristics lay a foundation for overcoming the drawbacks of traditional denoising methods and effectively suppress the generation of the pseudo-Gibbs phenomenon.

Assuming that the scale function and the wavelet $(\phi$, $\psi, \tilde{\phi}, \tilde{\psi})$ are designed by the filter $(h, g, \tilde{h}, \tilde{g})$, UDWT can efficiently decompose the input signal $c_{0}$ into $\left\{\omega_{1}, \cdots, \omega_{J}\right.$, $c_{J\}}$ through the following porous algorithm (àtrous) [19], where $\omega_{j}(j \in\{1,2, \cdots, \delta\})$ represents the wavelet coefficients on the scale $j$ and $c_{J}$ represents the wavelet coefficients on the coarsest resolution:

$$
\left\{\begin{array}{l}
c_{j+1}[l]=\left(\bar{h}^{(j)} * c_{j}\right)[l]=\sum_{k} h[k] c_{j}\left[l+2^{j} k\right] \\
\omega_{j+1}[l]=\left(\bar{g}^{(j)} * c_{j}\right)[l]=\sum_{k} g[k] c_{j}\left[l+2^{j} k\right]
\end{array}\right.
$$

when $l / 2^{j}$ is an integer, $h^{(j)}[l]=h[l]$, or $h^{(j)}[l]=0$; for example, $h^{(1)}=(\cdots, h[-2], 0, h[-1], 0, h[1], 0, h[2], \cdots), c_{j}$ can be reconstructed by 


$$
c_{j}[l]=\left(\tilde{h}^{(j)} * c_{j+1}\right)[l]+\left(\tilde{g}^{(j)} * \omega_{j+1}\right)[l]
$$

Since there is no downsampling process, the filter banks $(h, g, \tilde{h}, \tilde{g})$ only need to satisfy the complete reconstruction conditions in (3) and do not need to satisfy the de-aliasing conditions.

$$
\hat{h}^{*}(v) \widehat{\widetilde{h}}(v)+\hat{g}^{*}(v) \widehat{\tilde{g}}(v)=1
$$

Furthermore, the above porous algorithm can be extended to two-dimensional image decomposition in the following two-dimensional tensor product form:

$$
\begin{cases}c_{j+1}[k, l]=\left(\bar{h}^{(j)} \bar{h}^{(j)} * c_{j}\right)[k, l], & w_{j+1}^{1}[k, l]=\left(\bar{g}^{(j)} \bar{h}^{(j)} * c_{j}\right)[k, l] \\ w_{j+1}^{2}[k, l]=\left(\bar{h}^{(j)} \bar{g}^{(j)} * c_{j}\right)[k, l], & w_{j+1}^{3}[k, l]=\left(\bar{g}^{(j)} \bar{g}^{(j)} * c_{j}\right)[k, l]\end{cases}
$$

where $h g * c$ is the convolution performed with separable filters $h g$, which means being convolved with $h$ by column and then with $g$ by row. Three detailed scale images $w^{1}, w^{2}$, and $w^{3}$ are obtained at each scale, which have the same size as the original image.

To study the distribution of noise coefficients of the noisy images after UDWT transform, we use the maximum likelihood estimation (ML) method shown in (5) to obtain the variance estimation of each noisy observation sub-band [20]:

$$
\hat{\sigma}_{y}^{2}=\frac{1}{n \times n} \sum_{i} y^{2}(i)
$$

where $n \times n$ is the size of sub-band and $y$ is the observed image.

In this paper, Tank, Elaine, and Sea $(512 \times 512,8$ bpp $)$ are used as test images (see Fig. 1); Gaussian white noise with a mean value of 0 and variance of $30 \mathrm{~dB}$ and $50 \mathrm{~dB}$ are added using MATLAB. At the same time, three-layer UDWT decomposition is performed for the original image and noisy image, and three high-frequency sub-bands and one low-frequency sub-band are obtained. The standard deviation statistics about the low-frequency sub-bands and high-frequency sub-bands of the two kinds of images are given in Table 1.

As seen in Table 1, after decomposition by UDWT, the noise mainly distributes in the coefficients of the highfrequency sub-bands and the standard deviation of the low-frequency sub-band coefficients of the noisy images, and the images without noise are very close. That is, the influence of noise on the low-frequency sub-band of UDWT coefficients is very small. Hence, in the process of improved PDE model image denoising based on UDWT, we only need to deal with the high-frequency sub-band and keep the low-frequency sub-band state unchanged. In this way, we can improve the computation efficiency of diffusion and avoid the tendency of "over-strangling" the approximation sub-band coefficients.

\subsection{Proposed PDE model of the convexity-preserving diffusion function}

2.2.1 Analysis of $Y-K$ model

For a continuous functional in the region $\Omega$,

$$
E(u)=\int_{\Omega} f\left(\left|\nabla^{2} u\right|\right) d x d y
$$

where $\nabla^{2}$ is the Laplace operator, and when $f(\cdot) \geq 0$ and $\dot{f}(\cdot)>0$ is satisfied, $E(u)$ reaches its minimum value. The minimum is considered as a variational minimum problem, and the Euler-Lagrange equation of (6) could be obtained by the gradient descent method.

$$
\nabla^{2}\left(g\left(\left|\nabla^{2} u\right|\right) \nabla^{2} u\right)=0
$$

where $g(x)=f(x) / x$. According to this, You and Kaveh proposed the Y-K model [4] as (8)

$$
\left\{\begin{array}{l}
\frac{\partial u(x, y, t)}{\partial t}=-\nabla^{2}\left(g\left(\left|\nabla^{2} u\right|\right) \nabla^{2} u\right) \\
u(x, y, 0)=u_{0}(x, y)
\end{array}\right.
$$

where $\nabla^{2} u=\frac{\partial^{2} u}{\partial x^{2}}+\frac{\partial^{2} u}{\partial y^{2}}, u_{0}(x, y)$ is the original image,

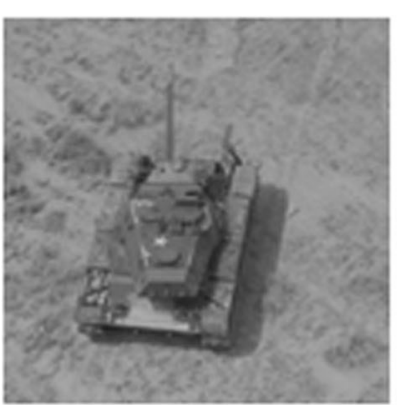

(a) Tank

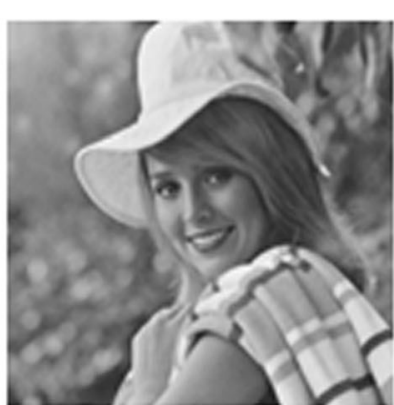

(b) Elaine

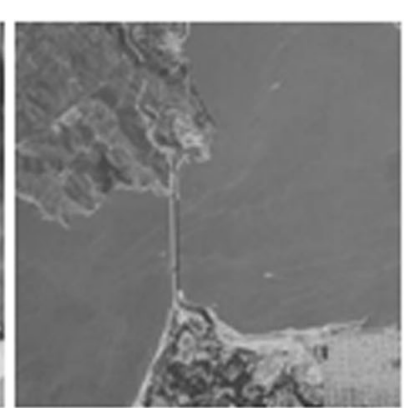

(c) Sea

Fig. 1 Three test images. a Tank. b Elaine. c Sea 
Table 1 The standard deviation estimate of each sub-band coefficients

\begin{tabular}{|c|c|c|c|c|c|c|c|c|c|c|}
\hline \multirow{3}{*}{$\begin{array}{l}\text { Test } \\
\text { image }\end{array}$} & \multirow{3}{*}{$\begin{array}{l}\text { Noiseless } \\
\text { height } \\
\text { frequency } \\
\text { sub-band } \\
\text { standard } \\
\text { deviation }\end{array}$} & \multicolumn{4}{|c|}{ High-frequency noisy sub-band } & \multirow{3}{*}{$\begin{array}{l}\text { Noiseless } \\
\text { low- } \\
\text { frequency } \\
\text { sub-band } \\
\text { standard } \\
\text { deviation }\end{array}$} & \multicolumn{4}{|c|}{ High-frequency noisy sub-band } \\
\hline & & \multicolumn{2}{|c|}{$30 \mathrm{~dB}$ noise } & \multicolumn{2}{|c|}{$50 \mathrm{~dB}$ noise } & & \multicolumn{2}{|c|}{$30 \mathrm{~dB}$ noise } & \multicolumn{2}{|c|}{$50 \mathrm{~dB}$ noise } \\
\hline & & $\begin{array}{l}\text { Standard } \\
\text { deviation }\end{array}$ & Disparity & $\begin{array}{l}\text { Standard } \\
\text { deviation }\end{array}$ & Disparity & & $\begin{array}{l}\text { Standard } \\
\text { deviation }\end{array}$ & Disparity & $\begin{array}{l}\text { Standard } \\
\text { deviation }\end{array}$ & Disparity \\
\hline \multirow[t]{3}{*}{ Tank } & 6.341 & 8.946 & 2.605 & 12.372 & 6.031 & 134.649 & 134.648 & 0.001 & 134.764 & 0.115 \\
\hline & 5.973 & 14.507 & 8.534 & 22.912 & 16.939 & & & & & \\
\hline & 5.494 & 27.205 & 21.711 & 44.715 & 39.221 & & & & & \\
\hline \multirow[t]{3}{*}{ Elaine } & 7.684 & 9.956 & 2.272 & 13.203 & 5.519 & 143.211 & 143.21 & 0.001 & 143.521 & 0.31 \\
\hline & 4.912 & 14.125 & 9.213 & 22.777 & 17.865 & & & & & \\
\hline & 5.553 & 27.301 & 21.748 & 44.828 & 39.275 & & & & & \\
\hline \multirow[t]{3}{*}{ Sea } & 7.218 & 9.581 & 2.363 & 12.911 & 5.693 & 119.241 & 119.246 & 0.005 & 119.429 & 0.188 \\
\hline & 7.264 & 15.101 & 7.837 & 23.332 & 16.068 & & & & & \\
\hline & 6.142 & 27.345 & 21.203 & 44.913 & 38.771 & & & & & \\
\hline
\end{tabular}

$u(x, y, t)$ is the image after smoothing, and $u_{0}(x, y)$ is in the time scale $t$, and we select $g(s)=\frac{1}{1+(s / k)^{2}}$ as diffusion coefficient, where $k$ is the edge threshold (constant).

The Y-K model reduces the block effect of low-order PDE models in the image denoising process and achieves a good balance between removing noise and preserving edges. However, in the process of denoising, the $\mathrm{Y}-\mathrm{K}$ model will produce a "speckle" effect, which leads to secondary contamination of the image. The reasons are as follows:

The energy functional $E(u)$ of (6) has the same concavity and convexity with $f($.$) , and if f($.$) is a convex func-$ tion, we can know $f^{\prime}(s) \geq 0$ and $f^{\prime}(s) \geq 0$, when $\forall s>0$. In this condition, $E(u)$ has a globally unique minimum value. However, in the Y-K model, $f(\cdot)$ is determined by the selected diffusion function $g(s)$ through $f(s)=s g(s)$. Overall, we obtain

$$
\left\{\begin{array}{l}
f^{\prime}(s)=\frac{s}{1+(s / k)^{2}} \\
f^{\prime \prime}(s)=\frac{k^{2}\left(k^{2}-s^{2}\right)}{\left(k^{2}+s^{2}\right)^{2}}
\end{array}\right.
$$

Clearly, $f^{\prime \prime}(s)<0$ when $s>k$, which means $f(\cdot)$ is no longer guaranteed to be convex. Therefore, it is not guaranteed that $E(u)$ has a globally unique minimum solution.

\subsubsection{Construction of the model}

For the nonconvexity of the diffusion function in the Y-K model, a new convexity-preserving diffusion function is constructed. Considering that the functional purpose of the diffusion function in the Y-K model is to detect edge information in images and that the Laplace operator $\nabla^{2}$ is very sensitive to noise, it is difficult to detect edge information effectively in noisy images. For this reason, we combine the
Gaussian filter with the Laplace operator, and Gaussian smoothing is performed before edge detection using the constructed convexity-preserving diffusion function. Based on this smoothing, an image diffusion denoising model (named Con_G\&L) is proposed, combining the constructed convexity-preserving diffusion function with the Gaussian filter and Laplace operator. The specific form is

$$
\left\{\begin{array}{l}
\frac{\partial u(x, y, t)}{\partial t}=-\nabla^{2}\left(g_{\text {Convex }}\left(\left|\nabla^{2}\left(G_{\sigma} \otimes u\right)\right|\right) \nabla^{2} u\right) \\
u(x, y, 0)=u_{0}(x, y)
\end{array}\right.
$$

where $G_{\sigma}(\cdot)=\frac{1}{\sqrt{2 \pi \sigma}} \exp \left(-\frac{|\cdot|^{2}}{2 \sigma^{2}}\right)$ is a Gaussian convolution kernel with variance $\sigma(\sigma>0), \otimes$ is the two-dimensional convolution operation, $\nabla^{2}$ is the Laplace operator, $u_{0}(x$, $y)$ is the original image, and $u(x, y, t)$ is the image after smoothing in the time scale $t, g_{\text {Convex }}(\cdot)$ is the diffusion function proposed whose specific form is

$$
g_{\text {Convex }}(s)=\frac{1}{\sqrt{1+(s / k)^{2 p}}}, p \in(0,1], s>0 .
$$

Here, $k$ is the threshold parameter to distinguish the edges and smooth areas of the image. If the value of $k$ is too small to distinguish the noise points well, it is prone to a step effect. If the value of $k$ is too large, the image will be blurred by excessive denoising. To increase the locally adaptive property, according to [21], $k$ is set as $k=1.4826 \times$ median $\left\|\left(\left|\nabla^{2} u\right|-\operatorname{median}\left(\left|\nabla^{2} u\right|\right)\right)\right\|$.

Furthermore, $g(s)$ is a nonnegative monotonously decreasing function, which satisfies

$$
g_{\text {Convex }}(0)=1, \quad \lim _{s \rightarrow \infty} g_{\text {Convex }}(s)=0 .
$$

In addition, $p \in(0,1]$ is the regulatory factor. The larger the $p$ is, the faster the function $g_{\text {Convex }}(s)$ decreases. Then, 
the better the edge information is preserved, the less the noise near the edges is removed. By contrast, the smaller $P$ is, the slower the function $g_{\text {Convex }}(s)$ decreases. Then, the worse the edge information is preserved, the stronger the noise near the edges is removed. Figure 2 shows the diagram of $g_{\text {Convex }}(\cdot)$ with $s$ when $k$ is 1 and $P$ is $1,0.9,0.7$, and 0.5 .

Effectiveness analysis of the Con_G\&L model is as follows:

Theorem. Based on the diffusion function $g_{\text {Convex }}(s)$, the energy functional $E(u)=\int_{\Omega} f\left(\left|\nabla^{2} u\right|\right) d x d y$ has a globally unique minimum value.

Proof: For the energy functional $E(u)=\int_{\Omega} f\left(\left|\nabla^{2} u\right|\right) d x d y$, $f($.) has the same concave-convex quality. According to the constructed diffusion function $g_{\text {Convex }}(s)$, we set $f(\cdot)$ as

$$
\left\{\begin{array}{l}
\frac{\partial u(x, y, t)}{\partial t}=-\nabla^{2}\left(g\left(\left|\nabla^{2} u\right|\right) \nabla^{2} u\right) \\
u(x, y, 0)=u_{0}(x, y)
\end{array}\right.
$$

Furthermore, we can obtain

$$
f^{\prime \prime}(s)=\frac{1-p}{\left(1+(s / k)^{2 p}\right)^{3 / 2}}
$$

due to $p \in(0,1]$, we can know $f^{\prime}$ ' $(x) \geq 0$. Thus, $f(s)$ is a convex function, and $E(u)$ has a globally unique minimum value.

\subsubsection{Discretization of the Con_G\&L model}

In image processing, an image is usually defined as a rectangular field $\Omega$, and it satisfies the following rectangular network in $\Omega$ :

$$
\left\{\begin{array}{c}
x=i \Delta x, \quad i=0,1, \cdots, I-1 \\
y=j \Delta y, \quad j=0,1, \cdots, J-1
\end{array}\right.
$$

where $I \times J$ is the size of an image, and we usually select $\Delta x=1, \Delta y=1$ for an image.

Let $u(\cdot, \cdot)$ be the processed image, $u_{0}(\cdot, \cdot)$ be the original image, $\Delta t$ be the time step, $h$ be the space step, $k$ be the iteration count in this condition, and mark $u(i, j)=u_{i, j}, u_{0}(i, j)=u_{i, j}^{0}$. Taking account of the accuracy of calculation, the central difference method is adopted in numerical calculation. The specific calculation format is

$$
\left\{\begin{array}{l}
\left(u_{x x}\right)_{i, j}^{k}=\frac{u_{i+1, j}^{k}-2 u_{i, j}^{k}+u_{i-1, j}^{k}}{h^{2}} \\
\left(u_{y y}\right)_{i, j}^{k}=\frac{u_{i, j+1}^{k}-2 u_{i, j}^{k}+u_{i, j-1}^{k}}{h^{2}} \\
\nabla^{2} u_{i, j}^{k}=\left(u_{x x}\right)_{i, j}^{k}+\left(u_{y y}\right)_{i, j}^{k} \\
\nabla^{2}\left(G_{\sigma} \otimes u\right)_{i, j}^{k}=\left(\left(G_{\sigma} \otimes u\right)_{x x}\right)_{i, j}^{k}+\left(\left(G_{\sigma} \otimes u\right)_{y y}\right)_{i, j}^{k}
\end{array}\right.
$$

Let

$$
\left\{\begin{array}{l}
c\left(\nabla^{2} u\right)=g_{\text {Convex }}\left(\left|\nabla^{2}\left(G_{\sigma} \otimes u\right)\right|\right) \nabla^{2} u \\
c_{i, j}^{k}=c\left(\nabla^{2} u_{i, j}^{k}\right)
\end{array},\right.
$$

we have

$$
\nabla^{2} c_{i, j}^{k}=\frac{c_{i+1, j}^{k}+c_{i-1, j}^{k}+c_{i, j+1}^{k}+c_{i, j-1}^{k}-4 c_{i, j}^{k}}{h^{2}}
$$

Then, the explicit difference scheme of the proposed Con_G\&L model is

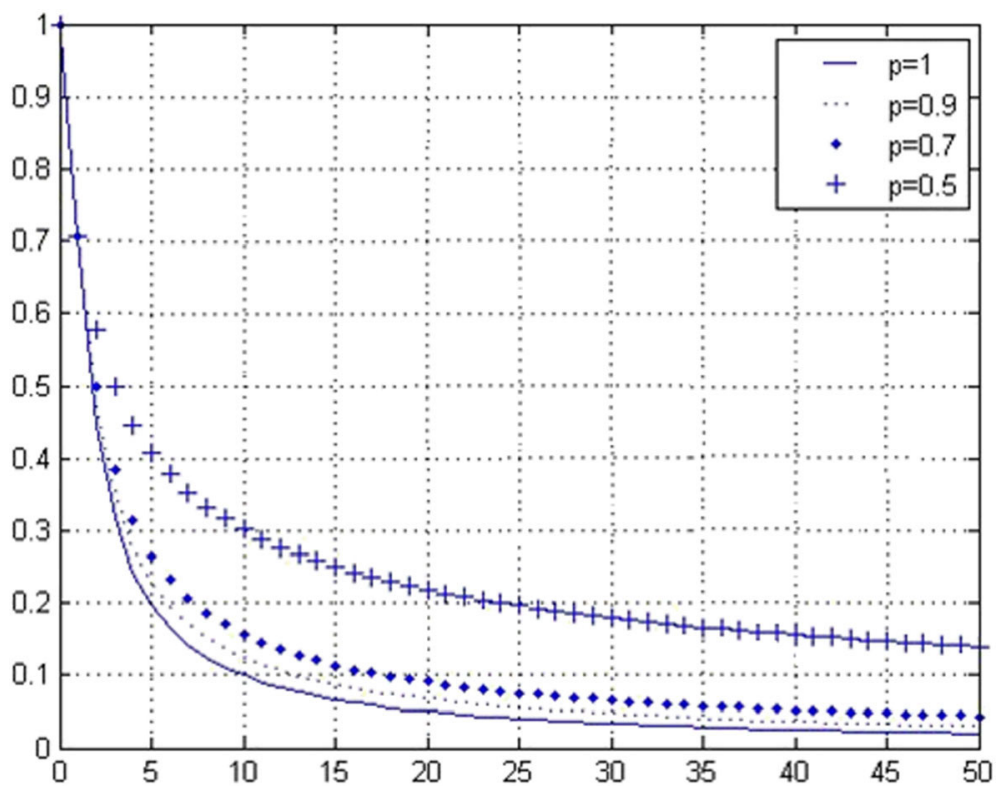

Fig. 2 The diagram of $g_{\text {Convex }}(s)$ when $k=1$ 


$$
u_{i, j}^{k+1}=u_{i, j}^{k}-\Delta t \nabla^{2} c_{i, j}^{k}
$$

\subsection{UDWT image denoising algorithm based on the Con_G\&L model}

From the analysis and discussion in Section 2.1, it can be seen that noise information is mainly contained in high-frequency sub-bands after UDWT decomposition of the noisy images. Based on that, this paper decomposes the noisy image into three layers via UDWT and then denoises the three high-frequency sub-bands using the proposed Con_G\&L model, while the low-frequency sub-band information remains unchanged. Finally, the final denoised image is obtained by reconstructing the low-frequency sub-band and the denoised highfrequency sub-band. The flow chart of the algorithm is shown in Fig. 3.

The specific implementation process of the algorithm is as follows:

Step 1. Deal with the noisy images via UDWT.

Step 2. Denoise the high-frequency sub-band components by using the Con_G\&L model after UDWT

decomposition.

Step 2.1 calculates ${ }^{2}\left(G_{\sigma} \quad u\right)$ of the high-frequency sub-band and then calculates $g_{\text {Convex }}\left(\left|{ }^{2}\left(G_{\sigma} \quad u\right)\right|\right)$ according to formula (11).

Step 2.2. Diffuse the Con_G\&L model according to the discrete form (16)-(19).
Step 2.3. If $\left|u_{i, j}^{k+1}-u_{i, j}^{k}\right|<0.01$, proceed to Step 3; otherwise, return to Step 2.1 .

Step 3. Apply the inverse UDWT transform for the highfrequency sub-band components after diffusion, which combines the low-frequency sub-band components; then, we obtain the denoised images.

\section{Results and discussions}

To verify the effectiveness of the proposed algorithm, a large number of simulation experiments have been carried out in this paper. The experiment was done with MATLAB (R2018a). The test images are "Elaine," "Tank, " "Sea," "Plane," and "Panzer" with size $512 \times 512$. In our experiments, the test images are corrupted by simulated additive noise with a standard deviation equal to 20, 30, 40, 50, and 60, respectively. Besides, we compare our model with the UDWT threshold, LLT, Y-K, and the denoising method proposed in [22]. The images are decomposed by three-layer UDWT (filter is $\mathrm{db} 9 / 7$ wavelet), the space step $h$ is 1 , the time step $\Delta t$ is 0.2 , and $P$ is 1 . Also, PSNR is used as the objective evaluation index of denoising effect:

$$
\operatorname{PSNR}=10 \times \lg \frac{255^{2} \times m \times n}{\sum_{i=1}^{m} \sum_{j=1}^{n}\left(u^{*}(i, j)-u^{0}(i, j)\right)^{2}}
$$

where $u^{*}(\cdot, \cdot)$ is the denoised image, $u^{0}(\cdot, \cdot)$ is the

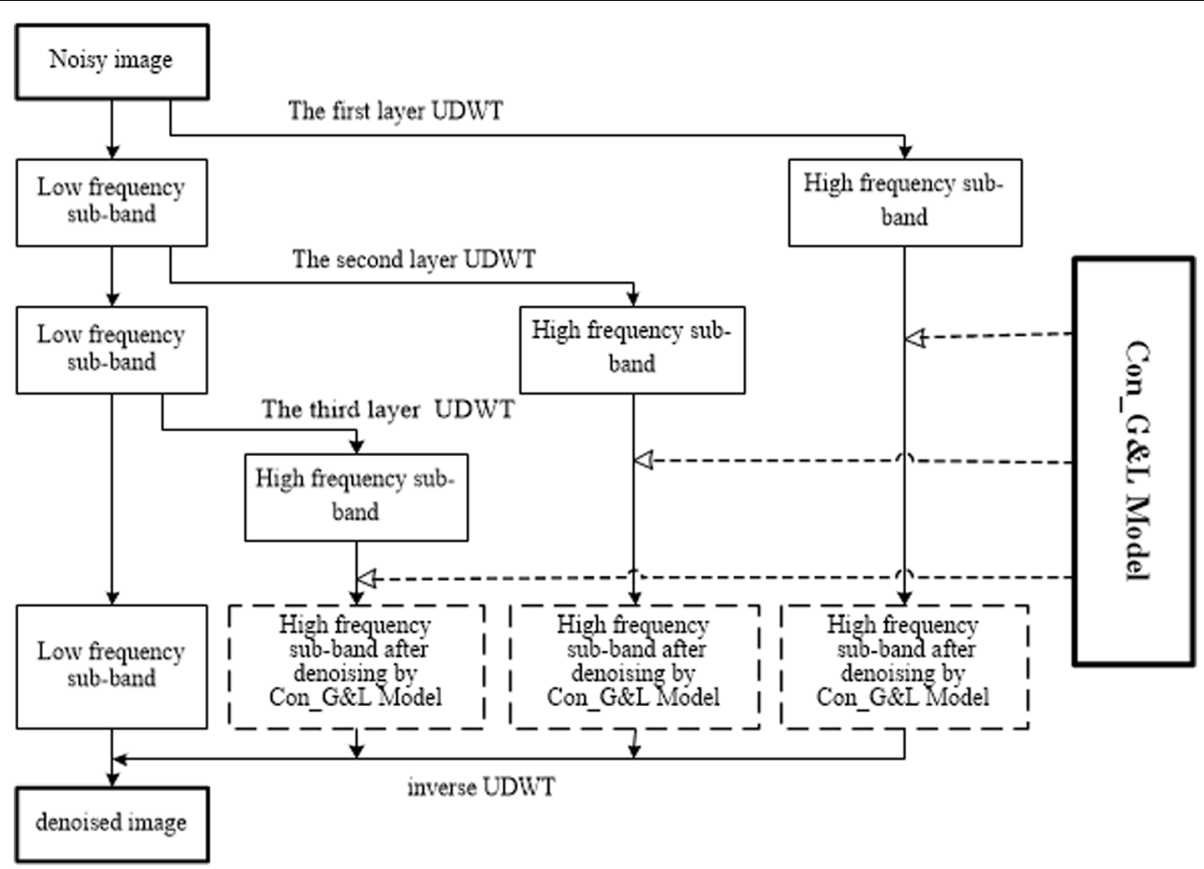

Fig. 3 Algorithm flow chart 
noiseless original image, $m$ is the length of the image, and $n$ is the width.

Figure 4 shows the denoising results of five test images after adding Gaussian white noise with a variance of 30 . Figure 5 is the denoising results of a local region of "Sea" when enlarging two times.

Table 2 shows the PNSR statistical results for the five denoised images of the algorithm in this paper and UDWT threshold method, LLT model, Y-K model, and the denoising method proposed in [22]. All the images are corrupted with Gaussian white noise with variances of $20,30,40,50$, and 60 before denoising.

It can be observed from Figs. 4 and 5 that, compared with the UDWT threshold method, LLT model method, Y-K model method, and the denoising method proposed in [22], the proposed method is better at maintaining the image texture, edges and details of the information, while removing noise. It can be seen from Table 2 that our method achieves superior evaluation indexes in most situations; proposed algorithm has higher PSNR than the other four denoising methods through comparing PSNR values in Table 2, especially for high variance noise.

\section{Conclusions}

As a typical representative of the fourth-order PDE model, the Y-K model can effectively suppress the "blocky" effect produced by the second-order PDE model in the process of denoising and achieves a good balance between image denoising and edge preservation. However, the nonconvexity of the diffusion function in the model makes it impossible for the energy function to have a globally unique minimum solution, which results in the "speckle" effect in the process of denoising. In addition, the Laplace operator used in the model is
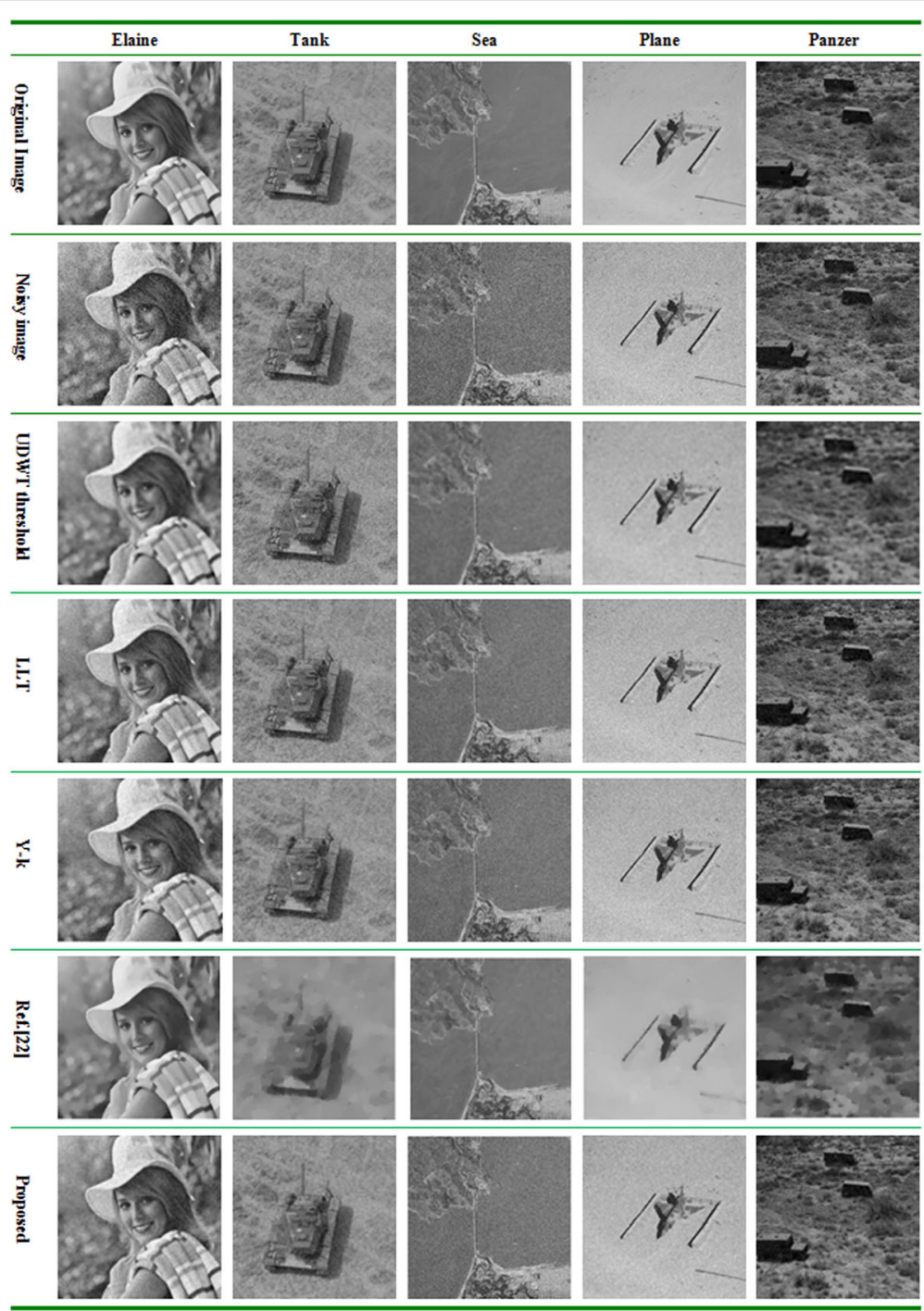

Fig. 4 The comparison of the denoising results for the test images 


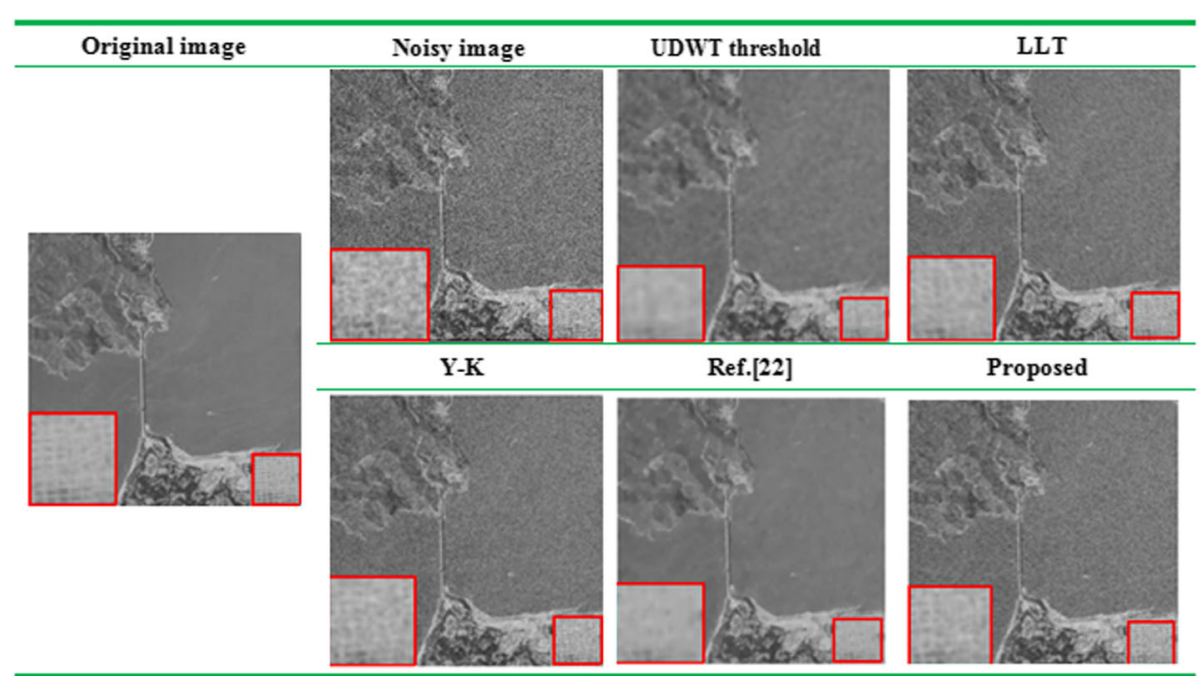

Fig. 5 The comparison of the denoising results of a local region when enlarging two times

Table 2 The PSNR of denoising for images of different methods

\begin{tabular}{|c|c|c|c|c|c|c|c|}
\hline \multirow[t]{2}{*}{ Image } & \multirow{2}{*}{$\begin{array}{l}\text { Noise } \\
\text { variance }\end{array}$} & \multicolumn{5}{|l|}{ PSNR (dB) } & \multirow[t]{2}{*}{ Proposed } \\
\hline & & Noisy image & UDWT threshold & LLT & $Y-K$ & Ref .[22] & \\
\hline \multirow[t]{5}{*}{ Elaine } & 20 & 22.13 & 29.61 & 29.84 & 29.64 & 30.15 & 30.22 \\
\hline & 30 & 18.62 & 28.67 & 28.15 & 28.01 & 28.94 & 29.34 \\
\hline & 40 & 16.11 & 27.77 & 27.21 & 25.86 & 28.2 & 28.35 \\
\hline & 50 & 14.13 & 27.09 & 24.54 & 23.86 & 27.18 & 27.28 \\
\hline & 60 & 12.57 & 26.63 & 23.11 & 21.52 & 27.23 & 26.71 \\
\hline \multirow[t]{5}{*}{ Tank } & 20 & 22.11 & 28.47 & 28.24 & 28.97 & 27.42 & 29.37 \\
\hline & 30 & 18.57 & 27.93 & 27.23 & 28.41 & 26.18 & 28.64 \\
\hline & 40 & 16.09 & 27.48 & 24.95 & 27.18 & 25.62 & 27.86 \\
\hline & 50 & 14.13 & 26.85 & 22.57 & 25.52 & 24.88 & 26.96 \\
\hline & 60 & 12.58 & 25.98 & 20.31 & 23.35 & 24.12 & 26.17 \\
\hline \multirow[t]{5}{*}{ Sea } & 20 & 22.09 & 28.19 & 27.72 & 27.87 & 29.25 & 28.29 \\
\hline & 30 & 18.61 & 27.63 & 27.01 & 27.48 & 27.77 & 27.79 \\
\hline & 40 & 16.09 & 26.82 & 24.93 & 25.56 & 26.86 & 27.06 \\
\hline & 50 & 14.16 & 26.18 & 22.62 & 23.63 & 26.14 & 26.31 \\
\hline & 60 & 12.59 & 25.34 & 20.36 & 21.69 & 25.39 & 25.46 \\
\hline \multirow[t]{5}{*}{ Plane } & 20 & 22.12 & 31.67 & 30.39 & 31.45 & 29.76 & 31.87 \\
\hline & 30 & 18.61 & 30.51 & 28.21 & 28.57 & 28.85 & 30.65 \\
\hline & 40 & 16.07 & 29.12 & 25.48 & 25.87 & 27.97 & 29.31 \\
\hline & 50 & 14.14 & 28.01 & 22.81 & 23.69 & 26.88 & 28.21 \\
\hline & 60 & 12.58 & 26.91 & 20.55 & 21.79 & 26.14 & 27.09 \\
\hline \multirow[t]{5}{*}{ Panzer } & 20 & 22.12 & 25.17 & 26.72 & 26.78 & 23.47 & 26.81 \\
\hline & 30 & 18.61 & 24.91 & 26.11 & 26.38 & 22.04 & 26.39 \\
\hline & 40 & 16.08 & 24.71 & 24.25 & 24.61 & 21.24 & 25.86 \\
\hline & 50 & 14.14 & 24.51 & 22.19 & 22.84 & 20.64 & 25.29 \\
\hline & 60 & 12.57 & 24.31 & 20.19 & 21.26 & 20.1 & 24.73 \\
\hline
\end{tabular}


hypersensitive to noise, making it difficult to detect edges in noisy images. Thus, the model loses the transformation and detail information of the image in the process of denoising. In this paper, we construct an image denoising model, Con_G\&L, based on a convexity-preserving diffusion function and Gaussian convolution. Then, the globally unique minimum solution of the energy functional is guaranteed by the convexity-preserving diffusion function, and the secondary pollution in the denoising process is avoided. At the same time, the ability to recognize details, such as edges in images, is improved by smoothing the Gaussian convolution. Then, an image denoising method based on UDWT and the Con G\&L model is proposed. In this method, the Con_G\&L model is applied to deal with the high-frequency sub-band of the UDWT in the noise image, which effectively suppresses the false edges and staircase effect. In short, this method removes the image noise effectively and maintains the image texture and other details at the same time.

\section{Abbreviations}

PDE: Partial differential equations; UDWT: Undecimated discrete wavelet transform

\section{Acknowledgements}

The authors would like to thank the editor and anonymous reviewers for their helpful comments and valuable suggestions.

\section{Authors' contributions}

XW conceived of the study and drafted the manuscript. WZ performed the statistical analysis and drafted the manuscript. RL carried out the design of the algorithm. RS carried out the comparative analysis on the research progress and the existing method. All authors read and approved the final manuscript.

\section{Funding}

This research has been funded by the National Natural Science Foundation of China (Grant Nos. 41671439, 41971388) and Innovation Team Support Program of Liaoning Higher Education Department (Grant No. LT2017013).

\section{Availability of data and materials}

The authors declare that the data and materials are available.

\section{Competing interests}

The authors declare that they have no competing interests.

\section{Author details}

${ }^{1}$ School of Geography, Liaoning Normal University, Dalian 116029, China. ${ }^{2}$ School of Computer and Information Technology, Liaoning Normal University, Dalian 116029, China. ${ }^{3}$ School of Mathematics, Liaoning Normal University, Dalian 116029, China.

Received: 4 April 2019 Accepted: 4 September 2019

Published online: 14 October 2019

\section{References}

1. C.G. Yan, H.T. Xie, J.J. Chen, A fast uyghur text detector for complex background images. IEEE Trans. Multimed. 20(12), 3389-3398 (2018)

2. C. Yan, L. Li, C. Zhang, B. Liu, Cross-modality bridging and knowledge transferring for image understanding. IEEE Trans. Multimed.. https://doi.org/ 10.1109/TMM.2019.2903448

3. C. Yan, Y.B. Tu, X.Z. Wang, STAT: Spatial-temporal attention mechanism for video captioning. IEEE Trans. Multimed.. https://doi.org/10.1109/TMM. 2019.2924576
4. A. Pandeyand, K.K. Singh, An overview of image denoising and image denoising techniques. Adv. Res. Electr. Electron. Eng. 2, 6-8 (2015)

5. G.A. Kumar, K. Kusagur, Evaluation of Image Denoising Techniques a Performance Perspective, International Conference on Signal Processing, Communication, Power and Embedded System (IEEE, Paralakhemundi, 2017), pp. 1836-1839

6. J.F. Sun, H.Y. Liu, Q. Caiand, A survey of image denoising based on wavelet transform. Boletín Técnico 55, 256-262 (2017)

7. P. Perona, J. Malik, Scale space and edge detection using anisotropic diffusion. IEEE Trans. Pattern Anal. Mach. Intell. 12(7), 629-639 (1990)

8. L. Alvarez, P.L. Lions, J.M. Morel, Image selective smoothing and edge detection by no-linear diffusion. SIAM J. Numer. Anal. 29(1), 182-193 (1992)

9. Y.L. You, M. Kaveh, Fourth-order partial differential equation for noise removal. IEEE Trans. Image Process. 9(10), 1723-1730 (2000)

10. M. Lysaker, A. Lundervold, C. Taix, Noise removal using fourth-order partial differential equation with applications to medical magnetic resonance images in space and time. IEEE Trans. Image Process. 12(12), 1579-1590 (2003)

11. T.W. Wang, J.Y. Wen, S.Y. Zhang, B.H. He, H.W. Luo, LLT denoising model based on fixed-point proximity algorithm. J. Jilin Univ.(Science Edition) 52(4), 794-796 (2014)

12. D.L. Donoho, I.M. Johnstone, G. Kerkyacharian, D. Picark, Wavelet shrinkage: asymptopia. J. R. Stat. Soc. Ser. 57, 301-369 (1995)

13. B. Vidakovic, Statistical modeling by wavelets. Wiley Series in Probability and Statistics: Applied Probability and Statistics. A Wiley-Interscience Publication. (Wiley, New York, 1999), ISBN: 0-471-29365-2

14. T.S. Qu, Y.S. Dai, S.X. Wang, Adaptive wavelet thresholding denoising method based on SURE estimation. Acta Electron. Sin. 30(2), 266-268 (2002)

15. F. Abramovich, T. Sapatinas, B.W. Sliverman, Wavelet thresholding via a bayesian approach [tech.rep.Bristol BS8 1TW] (1996)

16. H.X. Wang, A complex wavelet based spatially adaptive method for noised image enhancement. J. Comput. Aided Des. Comput. Graph 17(9), 1911-1916 (2005)

17. B. Fu, X.H. Wang, Image denoise algorithm based on inter correlation of wavelet coefficients at finer scale. Comput. Sci. 35(10), 246-249 (2008)

18. M.J. Shensa, Discrete wavelet transform: Wedding the a Trous and Mallat algorithms. IEEE Trans. Signal Process. 40(10), 2464-2482 (1992)

19. M.J. Shensa, The discrete wavelet transform: Wedding the àtrous and Mallatalgorithms. IEEE Trans. Signal Process 40(10), 2464-2482 (1992)

20. J.X. Li, P.L. Shui, Wavelet domain LMMSE-like denoising algorithm based on GGD ML estimation. J. Electron. Inf. Technol. 29(12), 2854-2857 (2007)

21. P.J. Rousseeuw, A.M. Leroy, Regression and Outlier Detection (Wiley, NewYork, 1987), pp. 39-46

22. S. Mukherjee, J. Farrand, W. Yao, A study of total-variation based noise reduction algorithms for low-dose cone-beam computed tomography. Int. J. Image Process. 10(4), 188-204 (2016)

\section{Publisher's Note}

Springer Nature remains neutral with regard to jurisdictional claims in published maps and institutional affiliations.

\section{Submit your manuscript to a SpringerOpen ${ }^{\circ}$ journal and benefit from:}

- Convenient online submission

- Rigorous peer review

- Open access: articles freely available online

High visibility within the field

- Retaining the copyright to your article

Submit your next manuscript at $>$ springeropen.com 